\title{
Quantum postulate vs. quantum nonlocality: on the role of the Planck constant in Bell's argument
}

\author{
Andrei Khrennikov ${ }^{1}$
}

Received: 9 April 2020 / Accepted: 9 November 2020 / Published online: 17 February 2021

(c) The Author(s) 2021

\begin{abstract}
We present a quantum mechanical (QM) analysis of Bell's approach to quantum foundations based on his hidden-variable model. We claim and try to justify that the Bell model contradicts to the Heinsenberg's uncertainty and Bohr's complementarity principles. The aim of this note is to point to the physical seed of the aforementioned principles. This is the Bohr's quantum postulate: the existence of indivisible quantum of action given by the Planck constant $h$. By contradicting these basic principles of QM, Bell's model implies rejection of this postulate as well. Thus, this hidden-variable model contradicts not only the QM-formalism, but also the fundamental feature of the quantum world discovered by Planck.
\end{abstract}

Keywords Complementarity and uncertainty principles · Indivisible quantum of action · Quantum postulate · Quantum nonlocality · Hidden variables · Bell inequality $\cdot$ Realism

\section{Introduction}

The aim of this paper is to show that the Bell's hidden-variable model [1-4] contradicts to the Bohr's quantum postulate [5-10]- the existence of indivisible quantum of action $h$. As is well known, Planck's discovery was the first step towards development of quantum theory. In particular, the first contribution of Bohr to quantum mechanics (QM), the principle of quantization of electron's orbits in an atom, was based on Planck's discovery of discreteness of action. Then, Bohr formalized the presence of indivisible quantum of action in nature as a special postulate, see Sect. 6 for details. The quantum postulate plays the basic role in quantum foundations. By rejecting it, one immediately confronts the problems of physics at the beginning of 20th century, including the problem of black

Andrei Khrennikov

andrei.khrennikov@lnu.se

1 International Center for Mathematical Modeling in Physics and Cognitive Sciences, Linnaeus

University, 35195 Växjö, Sweden 
body radiation. Thus, nobody is ready to reject explicitly the quantum postulate (the existence of $h$ ). However, in quantum foundational studies, this postulate is often rejected implicitly. The aim of this paper is to show that Bell's hidden-variable model implies rejection of the quantum postulate. One of the main motivations for Bell's study was to explain the long distance correlations (whatever "explain" means). In the light of our analysis, the following question arises:

Would one like to "explain" the long distance correlations by the cost of confronting again with the ultraviolet (Rayleigh-Jeans) catastrophe and ignoring the original Planck work on the black body radiation as well as the Einstein work on the photoelectric effect?

This paper is a part of the series of my works against nonlocal interpretation of QM [11-14]. The main message of aforementioned papers is that quantum theory is local. One of complications in criticizing "quantum nonlocality" is that it has two faces [15]:

- Apparent nonlocality of QM based on the projection postulate and discussed in the EPR-paper [16];

- Nonlocality of the Bell's hidden-variable model based on misleading interpretation of violation of the Bell inequalities.

In paper [11], violation of the Bell inequalities was treated in the purely quantum framework, i.e., without coupling to hidden variables (see also [17-28] and references in [17]):

What does quantum theory say about (non)violation of the Bell inequalities?

As was demonstrated in [11], violation vs. satisfaction of such inequalities is equivalent to local incompatibility vs. compatibility of observables. These inequalities should be treated as statistical tests for the complementarity principle. In the mathematical formalism, this is expressed in the form of noncommutativity of Hermitian operators representing local quantum observables.

This role of noncommutativity of quantum observables was highlighted already in the pioneer works of Griffiths [17,21] defending quantum locality (although not in such an explicit form as in [11] - through commutators of local observables). Griffiths wrote [17], chapter 24: "The first and most basic of these assumptions [in the derivation of Bell inequalities] is the existence of hidden variable with a mathematical structure which differs from the Hilbert space used in standard quantum mechanics.”

However, one can say that, in the Bell's framework, the genuine quantum approach to the Bell inequalities and, in particular, the mathematical structure of QM ([17-28, 11-14]) are not interesting. The essence of these inequalities is in their derivation on the basis of the Bell's hidden-variable model [1-4] and searching for explanation of precise quantum correlations (cf. with critical studies [29-35]). In this paper, we question this position by showing that the Bell model contradicts not only to the mathematical formalism of QM and its basic epistemic principles (uncertainty and complementarity), but also to the basic feature of the quantum physical world-the quantum postulate. 
During his life Bohr presented a variety of versions of the complementarity principle. In papers [13, 15, 36], I expressed my vision on Bohr's ideas as the block of sub-principles (see Sect. 7). I think that such a compact formulation of Bohr's principles is important for further discussions of the type "Bohr vs. Bell" [13]. Nowadays, the Bohr complementarity principle is discussed mainly by philosophers, e.g., in [37-41]. Since the complementarity principle has the nontrivial epistemic structure and since Bohr's writings are not so simple for understanding, we collect the basics of Bohr's views related to Bell's argumentation in Sect. 2. The reader interested in more details can continue reading in Sect. 7, but, in principle, Sect. 2 is sufficient for our aim.

Now, I shortly remind my argumentation against quantum nonlocality. In [11], it is was shown that violation of Bell-type inequalities-for quantum observablescan be mathematically formulated in terms of commutators of local observables. In QM, noncommutative observables are known as incompatible. Thus, violation of Bell's inequality - for quantum observables - is a consequence of the Bohr's complementarity principle. Therefore, even formulation of the Bell's hidden-variable model contradicts to the basic principles of QM (Bohr's complementarity and, in fact, Heisenberg's uncertainty principles). This was the output of paper [11]. However, in principle, one still can say:

What is the matter? May be we can violate these basic principles of $Q M$ and even abandon the mathematical formalism of $Q M$.

In this note, we emphasize that by abandoning the basic principles (complementarity and uncertainty) of QM, one confronts not only with the formalism of quantum theory, containing observables represented by noncommuting operators, but even with the most fundamental feature of quantum world, the existence of indivisible quantum of action $h$.

Thus, the aim of this paper is not to extend the noncommutativity argument against the Bell's nonlocality (it was done in papers [11-14] supporting conclusions of the previous works in this direction [17-28]). The main message of this paper is that noncommutativity is not simply a mathematical specialty of quantum theory, but that it is a consequence of the quantum postulate - the existence of the irreducible quantum of action.

\section{Bohr vs. Einstein and Bell}

The complete comparative analysis of the views of Bohr on quantum foundations versus the views of Einstein and later Bell can be found in monographs [37-41], see also the recent papers of Plotnitsky [42-44], especially the last one. Here we just briefly remind the key-points of this analysis, which are coupled to the present paper (see also Sect. 7). As was pointed in the report of one of paper's reviewers:

A particular aspect of Bohr's understanding of quantum mechanics is of special relevance in connection with Bell. What did Bohr have to say about the properties of a quantum system in isolation, when it is not being measured? In particular, how can one use the macroscopic outcome of a meas- 
urement to infer an earlier property of a microscopic quantum system just before it interacts with the measuring device? This is important, because addressing this matter seems to have been a significant motivation for Bell's work, as shown by his references to 'beables', and comments in one of his last papers, 'Against Measurement'.

Bohr's position was that QM is a theory of measurements. It does not say anything about a system in isolation, about so to say system's ontic state. Quantum state represents the previously performed measurement procedure leading to state preparation. Then, concerning the use of "the macroscopic outcome of a measurement to infer an earlier property of a microscopic quantum system just before it interacts with the measuring device", Bohr pointed (see [10]) that generally a quantum system has no physical properties that can be assigned it before measurement, because his view is that we cannot consider quantum systems separately from their interactions with measuring instruments. Both Einstein and Bell (and then Griffiths [17, 20-22, 25-27] and Ozawa [45]) believe that it should be possible to do so.

Plotnitsky described Bohr's position as follows (private communication): “Bohr's view of the quantum-mechanical situation may be described in this way. Consider a quantum object, say, an electron. It can never be observed by itself, without an essential interference by a measuring instrument, unlike in classical physics, can never be neglected or controlled so as to be able to compensate for it and treat the electron's behavior independently. But an electron can interact with a measuring instrument. While we cannot observe this interaction or control it either because it is quantum, which compels Bohr to speak, in his reply to EPR, of the "uncontrollable" nature of this interaction (Bohr 1935), this interaction has an effect on, leaves a trace in, the measuring instrument, say, in the case of a position measurement, a spot on the screen, an effect that is classical and that is observed or registered. This trace is a product of this interaction, and, in Bohr's view, never a manifestation of a pre-existing property (in the case a position) of the electron. Not is this trace is not a distorted value of the position of the electron before measurement (as it would be in Bohmian mechanics). It strictly an effect of this interaction. There is no position assignable to the electron before this interaction; at most one could speak of this position at the time of this interaction, as Bohr did in his earlier works, including in his reply to EPR, before around 1937-1938. There are further complexities, especially in Bohr's post-EPR thinking, to the effect that it may not even be possible to speak of an electron at from an event of measurement, but these complexities can be put aside here."

The above citation of the aforementioned reviewer has the following continuation: "Physicists who do experiments at accelerator laboratories, including the one where Bell worked, speak without hesitation about their measurements revealing properties (energy, momentum) possessed by particles just before they arrive at the detector. There is usually much less concern about the later history of the particle when the measurement is over; quite the reverse of what one finds in some textbooks and much of the quantum foundations literature. If Bohr (and the other Founding 
Fathers) could not address this problem, Bell should deserve some credit for trying, even if in the end he did not succeed." 1

Of course, a system can be prepared in a state such that a further measurement of say energy would give with probability one the concrete value of energy. But, even in this special case probability is involved, probability one does not mean always (see also [44]). But, generally by Bohr one cannot speak about preexisting properties, before measurement. Some people speak, but this is merely jargon; moreover, most of them speak of such properties at the time of measurements, which is very different and is more acceptable. ${ }^{2}$

One can be curious why Bohr claimed repeatably that it is impossible to infer an earlier property of a microscopic quantum system just before it interacts with the measuring device. Our aim is to justify Bohr's claim by the complementarity principle and at a deeper level by the quantum postulate. This justification leads to understanding that Bell's attempt to introduce hidden variables determining outputs of quantum measurements was really misleading, from the very beginning, i.e., disregard to derivation the Bell inequality. ${ }^{3}$

\section{Bell}

Consider Bell's random variables $A(a, \lambda), B(b, \lambda)$ representing observables of Alice and Bob, respectively. Here, as usual , $\lambda$ represents the state of a compound system, $\lambda$ belongs to some set $\Lambda$, its points are called hidden variables. The parameters $a$ and $b$ represent settings of observables of Alice and Bob, say orientations of polarization beam splitters. The set $\Lambda$ is endowed with a probability measure $p .{ }^{4}$ Surprisingly, Bell did not highlight that, besides the probabilities

$$
p_{a, b}(x, y)=p(\lambda \in \Lambda: A(a, \lambda)=x, B(b, \lambda)=y)
$$

for compatible observables, this model also describes the probabilities

\footnotetext{
${ }^{1}$ It is interesting that the position presented in the citation from the report is very common for people working in high energy physics. I had numerous conversation and my collocutors spoke freely, e.g., about the trajectories of quantum particles and so on. What is the reason for spread of such views in high energy physics? Just collective psychology? Or specialty of behavior of high energy particles?

2 As was pointed by Plotnitsky (again the private communication): "Bohr for while held such a view, including in his reply to EPR, but after 1937, when his position was that one cannot assign any properties to quantum objects even at the time of measurement. All physical properties considered are those of measuring instruments, and these properties can be described classically (subject to the uncertainty relations), but they cannot be predicted by means of classical physics."

3 Although in the EPR paper [16] Einstein and his coauthors considered the values before measurement, later Einstein changed his position. He considered classical field theory as the fundamental physical theory and QM as corresponding measurement theory [46]. Although Einstein spent the last years of his life by working on this project, he did not succeed to combine classical field theory and QM. An attempt to realize Einstein's dream was done in a series of papers of the author on so-called prequantum classical statistical field theory [47-49].

${ }^{4}$ In the rigorous mathematical framework, $\Lambda$ should be endowed with $\sigma$-algebra and $p$ is a countably additive measure on this $\sigma$-algebra. If set $\Lambda$ is finite, then we can consider algebra of all its subsets. For probability measure $p$, Bell used notation $d \rho(\lambda)$ or even $\rho(\lambda) d \lambda$.
} 


$$
\begin{aligned}
& p_{a, a^{\prime}}\left(x_{1}, x_{2}\right)=p\left(\lambda \in \Lambda: A(a, \lambda)=x_{1}, A(a, \lambda)=x_{2}\right), \\
& p_{b, b^{\prime}}\left(y_{1}, y_{2}\right)=p\left(\lambda \in \Lambda: B(b, \lambda)=y_{1}, B\left(b^{\prime}, \lambda\right)=y_{2}\right),
\end{aligned}
$$

for generally incompatible observables represented by noncommuting operators. This point was enlighten in Fine's paper [50] and repeated in various versions by many authors [29-35].

For quantum observables, even without any Bell's type inequality, Bell' hiddenvariable model contradicts to QM-representation of observables by Hermitian operators and, hence, to the Heisenberg uncertainty principle and more generally to the Bohr complementarity principle.

In principle, the Bell model can be considered as a metaphysical model, as a kind of pure theoretization on possible features of the subquantum world. But, Bell identified the values of subquantum quantities with the values of real physical observables described by QM (because he wanted the experimental verification of his approach). This identification immediately leads to confrontation with quantum theory. ${ }^{5}$

Thus, from the very beginning Bell's hidden-variable model was designed as contradicting the basic principles of QM, uncertainty and complementarity.

\section{Questioning the Pinciple of Complementarity}

The Heisenberg uncertainty principle was the starting point for Bohr's formulation of the complementarity principle [5-9] (see my recent papers [11, 13, 36] for nonphilosophers gently presentation of this principle, see also Sect. 7). Thus, in the light of above consideration, we can say that in fact Bell's argument was directed against the Bohr complementarity principle. This attack against complementarity was overshadowed by nonlocality issue.

We stress that rejection of the complementarity principle (or the Heisenberg uncertainty principle) would have similar catastrophic consequences even for noncompound systems, say a single atom or neutron, as we can see from the contextuality tests (see, e.g., [52]).

To discard the Bell's hidden-variable model, one needn't derive inequalities and test them experimentally. Of course, one should believe in the basic principles of QM.

The main impact of experimental tests [53-57] is demonstration that quantum correlations (predicted by QM) are preserved for long distances. The latter plays

\footnotetext{
5 Precisely this viewpoint on Bell's approach was emphasized by De Broglie [51] (for many years ago), who was very critical to Bell's modeling and did not take Bell's inequality seriously. We remark that generally De Broglie was not against the realistic interpretation of QM nor against nonlocality. But, he was against Bell's too straightforward coupling between subquantum and quantum theory; cf. with prequantum classical statistical field theory : a local theory with hidden variables (classical random fields), but without identification of the values of subquantum quantities - quadratic forms of random fields, with the values of quantum observables).
} 
the crucial role in quantum engineering. However, correlations preservation can be checked directly without inequalities. ${ }^{6}$

\section{Long Distance Correlations and Violation of Complementarity Principle}

Typically, followers of the Bell argument say that they want to explain the long distance correlations. I think that the essence of the problem is in the word "explain". In science, we operate with mathematical models of physical processes. So, "explain" means "to describe by some mathematical model". And quantum mathematics, as a mathematical model, describes perfectly the long distance correlations: entangled states and projection type measurements. So, it seems that Bell and his followers have something different in mind.

Why was Bell not satisfied with the quantum mechanical description?

From reading Bell, I have the impression that he "simply" wanted to re-establish realism of classical physics, to be able to operate with values of quantum observables before measurement. But, what is the main quantum barrier for such realism? Everybody knows this very well, this is the Bohr complementarity principle with starting point at the Heisenberg uncertainty relation; see Bohr [5]:

... an independent reality in the ordinary physical sense can neither be ascribed to the phenomena nor to the agencies of observation.

Suppose somebody, say Alice, questions the Heisenberg uncertainty principle. Then, why should she consider compound systems? Does she think that these principle is violated only for compound systems? It would be really strange. Thus, before trying to explain the long distance correlations with the Bell-type hiddenvariable model, it would be reasonable to try explain incompatibility of observables corresponding spin projections to different axes or incompatibility of position and momentum observables.

The main feature of the Bell's hidden-variable model, the feature crying for justification, is violation of the complementarity principle. It is not so natural to try to"explain" long distance correlations without any attempt to explain violation of this principle.

\footnotetext{
${ }^{6}$ Moreover, by operating with say CHSH-combination of correlations experimenter can miss mutual compensation of deviations from QM. In Aspect's pioneer experiment [58], correlations did not match the quantum prediction, but they mystically compensated each other to violate the Bell inequality (see [59] for discussion). In spite of numerous discussions with experimenters, I am still not sure that data from the basic experiments on say $\mathrm{CHSH}$-inequality is clean from the mentioned Aspect-type anomaly. Papers typically present only the $\mathrm{CHSH}$-correlation combination, but not separate correlations for pairs of experimental settings.
} 


\section{Quantum Postulate}

By going against complementarity, it is useful to remind its foundational roots. The Bohr's complementarity principle will be discussed in detail in Sect. 7. This principle is an epistemic statement, i.e., about extraction of knowledge about micro-systems with the aid of classical measurement apparatuses. Now we present the physical root of this principle.

For Bohr, the root of the complementarity is the existence of indivisible quantum of action given by the Planck constant $h$. The existence of this quantum prevents separation of the genuine properties of a system from the features of interaction with a measurement apparatus. So, the seed of the Bohr complementarity principle is the existence of the indivisible quantum of action. The latter was formulated in the form of the quantum postulate ([5], p. 580):

Its [quantum theory] essence may be expressed in the so-called quantum postulate, which attributes to any atomic process an essential discontinuity, or rather individuality, completely foreign to the classical theories and symbolised by Planck's quantum of action."

As was mentioned, Bohr's writings are not so simple for understanding; he presented his thoughts in very compressed form. A good decoding of Bohr's formulation of the quantum postulate can be found in paper [60]. We cite its beginning:

"Niels Bohr considered that the 'discovery of the elementary quantum of action, which revealed a feature of wholeness inherent in atomic processes' ([8], p. 2) means a departure from the classical physics description, where we assume that the interaction of a physical system with a measuring ultimately be disregarded. In the case of quantum phenomena we have to be aware that due to the 'indivisibility of the quantum of action' ([7], p. 5) we must associate to all 'individual atomic processes an element of discontinuity quite foreign to the fundamental principles of classical physics, according to which all actions may vary in a continuous manner' ([7], p. 5). This means that in the case of all atomic processes every energy change results from an indivisible - and because of that discontinuous - transition between different states that cannot be continuously connected. Bohr's ideas were summed up and presented in what he called the quantum postulate (and its consequences)."

We continue and cite Bohr again ([5], p. 580):

The quantum postulate implies that any observation of atomic phenomena will involve an interaction with the agency of observation not to be neglected ... the definition of the state of a physical system, as ordinarily understood, claims the elimination of all external disturbances. But in that case, according to the quantum postulate, any observation will be impossible ... if in order to make observation possible we permit certain interactions with suitable agencies of measurement, not belonging to the system, an unambiguous definition of the state of the system is naturally no longer possible."7

\footnotetext{
${ }^{7}$ It should be noted here that several authors, in particular, Griffiths [17, 20-22, 25, 26] and Ozawa [45] take a different position on this issue, by arguing that pre-measurement values can be consistently assigned to quantum observables in the QM-framework. While these are important arguments, it would not be possible to consider them in detail to do them justice within my limits in this article. The reader can find the detailed presentation of Griffiths' position in Stanford Encyclopedia article [27].
} 


\section{Bohr's Complementarity Principle}

In 1949, Bohr [10] presented the essence of complementarity in the following widely citing statement:

"This crucial point ... implies the impossibility of any sharp separation between the behavior of atomic objects and the interaction with the measuring instruments which serve to define the conditions under which the phenomena appear. In fact, the individuality of the typical quantum effects finds its proper expression in the circumstance that any attempt of subdividing the phenomena will demand a change in the experimental arrangement introducing new possibilities of interaction between objects and measuring instruments which in principle cannot be controlled. Consequently, evidence obtained under different experimental conditions cannot be comprehended within a single picture, but must be regarded as complementary in the sense that only the totality of the phenomena exhausts the possible information about the objects."

By analyzing this Bohr's statement, I propose $[11,13,36]$ to present the Bohr complementarity principle as the following four interconnected principles:

- Contextuality: Irreducible dependence of measurement's output on the experimental context.

- Context complementarity: Existence of complementary experimental contexts.

- Individuality: Discreteness of quantum measurements -generation of physical phenomena.

- Completeness: Complementary observations provide complete information about system's state.

In this formulation, the complementarity principle can be treated as an epistemological principle (see, especially, paper [36] on coupling to quantum information theory).

\section{Concluding Remarks}

The ontological seed of the complementarity principle is the quantum postulate. Rejection of complementarity, as was done by Bell in his hidden-variable model, is impossible without rejection of the existence of indivisible quantum of action, given by the Planck constant $h$. By rejection of the latter, we would cast away all physical achievements of 20th century and confront again the problem of black body radiation (and photo-electric effect).

During his life Bohr emphasized the irreducible role of measurement and the impossibility to assign to quantum systems the properties expressed by quantum observables. However, Bohr did not have the no-go attitude, cf. with the von Neumann [61] and Bell [1] no-go theorems. It seems (see [44]) that he did not reject 
the possibility that a more general physical theory would be created in future, theory of a realistic type (whatever the latter can mean). But, such a subquantum theory should explain the existence of the irreducible quantum of action in nature and it should be in peace with the complementarity principle. ${ }^{8}$

Funding Open access funding provided by Linnaeus University.

Open Access This article is licensed under a Creative Commons Attribution 4.0 International License, which permits use, sharing, adaptation, distribution and reproduction in any medium or format, as long as you give appropriate credit to the original author(s) and the source, provide a link to the Creative Commons licence, and indicate if changes were made. The images or other third party material in this article are included in the article's Creative Commons licence, unless indicated otherwise in a credit line to the material. If material is not included in the article's Creative Commons licence and your intended use is not permitted by statutory regulation or exceeds the permitted use, you will need to obtain permission directly from the copyright holder. To view a copy of this licence, visit http://creativecommons.org/licen ses/by/4.0/.

\section{References}

1. Bell, J.S.: On the Einstein-Podolsky-Rosen paradox. Physics 1, 195-200 (1964)

2. Bell, J.S.: Speakable and Unspeakable in Quantum Mechanics, 2nd edn. Cambridge University Press, Cambridge, UK (2004)

3. Bell, J.S.: On the problem of hidden variables in quantum theory. Rev. Mod. Phys. 38, 450 (1966)

4. Clauser, J.F., Horne, M.A., Shimony, A., Holt, R.A.: Proposed experiment to test local hidden-variable theories. Phys. Rev. Lett. 23, 880 (1969)

5. Bohr, N.: The quantum postulate and the recent development of atomic theory. Suppl. Nat. 121, 580-590 (1928)

6. Bohr, N.: Wirkungsquantum und Naturbeschreibung. Naturwissenschaft 17, 483-486 (1929)

7. Bohr, N.: Atomic Theory and the Description of Nature. Cambridge University Press, Cambridge (1934)

8. Bohr, N.: Causality and complementarity. in Essays 1958-1962 on atomic physics and human knowledge, pp. 1-7. Interscience, New York (1963)

9. Bohr, N.: The quantum of action and the description of nature. In: Kalckar, J. (ed.) Foundations of Quantum Physics I (1926-1932). Niels Bohr collected works, vol. 6, pp. 201-217. Elsevier B.V, New York (1985)

10. Bohr, N.: The Philosophical Writings of Niels Bohr. Ox Bow Press, Woodbridge, UK (1987)

11. Khrennikov, A.: Get rid of nonlocality from quantum physics. Entropy 21(8), 806 (2019)

12. Khrennikov, A.: Bell argument: Locality or realism? Time to make the choice. AIP Conf. Proc., 1424, pp. 160-175 (2012), https://arxiv.org/pdf/1108.0001v2.pdf

13. Khrennikov, A.: Bohr against Bell: complementarity versus nonlocality. Open Phys. 15, 734-73 (2017)

14. Khrennikov, A.: Quantum versus classical entanglement: eliminating the issue of quantum nonlocality. arXiv:1909.00267v1 [quant-ph]

15. Khrennikov, A.: Two faced Janus of quantum nonlocality. arXiv:2001.02977 [quant-ph]

\footnotetext{
8 Prequantum classical statistical field theory (PCSFT) [47-49], classical random field model for subquantum processes, seems to be of such type. However, in PCSFT connection between the subquantum processes and measurement is more complex than in Bell's hidden-variable model; the values of subquantum physical quantities, quadratic forms of random fields, cannot be straightforwadly identified with the values of quantum observables. In particular, the values of quantum observables cannot be assigned to physical systems—random fields.
} 
16. Einstein, A., Podolsky, B., Rosen, N.: Can quantum-mechanical description of physical reality be considered complete? Phys. Rev. 47, 777-780 (1935)

17. Griffiths, R.B.: Consistent Quantum Theory (Cambridge 2002). http://quantum.phys.cmu.edu/CQT/

18. De Muynck, W.: Foundations of Quantum Mechanics, An Empiricist Approach. Springer, Dordrecht (2006)

19. Khrennikov, A.Y.: The role of von Neumann and Luders postulates in the Einstein, Podolsky, and Rosen considerations: comparing measurements with degenerate and nondegenerate spectra. J. Math. Phys. 49(5), 52102 (2008)

20. Griffiths, R.B.: Quantum locality. Found. Phys. 41, 705-733 (2011)

21. Griffiths, R.B.: Consistent quantum measurements. Stud. Hist. Phil. Mod. Phys. 52, 188-197 (2015)

22. Griffiths, R.B.: What quantum measurements measure. Phys. Rev. A 96, 032110 (2017)

23. Boughn, S.: Making sense of Bell's theorem and quantum nonlocality. Found. Phys. 47, 640-657 (2017)

24. Jung, K.: Violation of Bell's inequality: Must the Einstein locality really be abandoned? J. Phys.: Conf. Ser. 880, 012065, 1-8 (2017)

25. Griffiths, R.B.: Quantum nonlocality: Myth and reality. arXiv 2019, arXiv:1901.07050

26. Griffiths, R.B.: Nonlocality claims are inconsistent with Hilbert-space quantum mechanics. Phys. Rev. A 101, 022117 (2020). arXiv: 1901.07050

27. Griffiths, R.B.: "The Consistent Histories Approach to Quantum Mechanics", Stanford Encyclopedia of Philosophy (2019) https://plato.stanford.edu/entries/qm-consistent-histories/

28. Cetto, A.M., Valdes-Hernandez, A., de la Pena, L.: On the spin projection operator and the probabilistic meaning of the bipartite correlation function. Found. Phys. 50, 27-39 (2020)

29. Kupczynski, M.: Bertrand's paradox and Bell's inequalities. Phys. Lett. A 121, 205-207 (1987)

30. Khrennikov, A.: Non-Kolmogorov probability models and modified Bell's inequality. J. Math. Phys. 41, 1768-1777 (2000)

31. Khrennikov, A., Yu.: Interpretations of Probability, VSP Int. Sc. Publishers: Utrecht/Tokyo, 1999, 2nd edn., De Gruyter: Berlin, (2009)

32. Nieuwenhuizen, T.M.: Is the contextuality loophole fatal for the derivation of Bell inequalities? Found. Phys. 41, 580-591 (2011)

33. De Raedt, H., Hess, K., Michielsen, K.: Extended Boole-Bell inequalities applicable to quantum theory. J. Comput. Theor. Nanosci. 8, 10119 (2011)

34. Khrennikov, A.: CHSH inequality: quantum probabilities as classical conditional probabilities. Found. Phys. 45, 711-725 (2015)

35. Khrennikov, A., Alodjants, A.: Classical (local and contextual) probability model for Bohm-Bell type experiments: no-signaling as independence of random variables. Entropy 21, 157 (2018)

36. Khrennikov, A.: Echoing the recent Google success: Foundational roots of quantum supremacy. arXiv:1911.10337 [quant-ph]

37. Jaeger, G.: Quantum Information. An Overview. Springer, Berlin (2007)

38. Plotnitsky, A.: Epistemology and Probability: Bohr, Heisenberg, Schrödinger and the Nature of Quantum-Theoretical Thinking. Springer, Berlin (2009)

39. Plotnitsky, A.: Niels Bohr and Complementarity: An Introduction. Springer, Berlin (2012)

40. Jaeger, G.: Quantum Objects: Non-Local Correlation, Causality and Objective Indefiniteness in the Quantum World. Springer, Berlin (2013)

41. Faye, J., Folse, H.: Niels Bohr and the Philosophy of Physics: Twenty-First-Century Perspectives. Bloomsbury Academic, London (2017)

42. Plotnitsky, A.: "Without in any way disturbing the system": Illuminating the issue of quantum nonlocality. arXiv:1912.03842 [quant-ph]

43. Plotnitsky, A.: Reality, indeterminacy, probability, and information in quantum theory. Entropy 22(7), 747 (2020). https://doi.org/10.3390/e22070747

44. Plotnitsky, A.: The unavoidable interaction between the object and the measuring instruments: reality, probability, and nonlocality in quantum physics. Phys, Found (2020). https://doi.org/10.1007/ s10701-020-00353-5

45. Ozawa, M.: Intersubjectivity of outcomes of quantum measurements. arXiv:1911.10893 [physics. gen-ph]

46. Einstein, A., Infeld, L.: The Evolution of Physics: The Growth of Ideas from Early Concepts to Relativity and Quanta. Cambridge University Press, Cambridge (1938)

47. Khrennikov, A.: A pre-quantum classical statistical model with infinite-dimensional phase space. J. Phys. A 38, 9051-9073 (2005) 
48. Khrennikov, A.: Beyond Quantum. Pan Stanford Publishers, Singapore (2014)

49. Khrennikov, A.: Detection model based on representation of quantum particles by classical random fields: Born's Rule and Beyond. Found. Phys. 39, 997-1022 (2009)

50. Fine, A.: Hidden variables, joint probability, and the Bell inequalities. Phys. Rev. Lett. 48, 291 (1982)

51. De Broglie, L.: The Current Interpretation of Wave Mechanics: A Critical Study. Elsevier, New York (1964)

52. Bartosik, H., Klepp, J., Schmitzer, C., Sponar, S., Cabello, A., Rauch, H., Hasegawa, Y.: Experimental test of quantum contextuality in neutron interferometry. Phys. Rev. Lett. 103, 040403 (2009)

53. Aspect, A., Dalibard, J., Roger, G.: Experimental test of Bell's Inequalities using time-varying analyzers. Phys. Rev. Lett. 49, 1804-1807 (1982)

54. Weihs, G., et al.: Violation of Bell's inequality under strict Einstein locality conditions. Phys. Rev. Lett. 81, 5039 (1998)

55. Hensen, B., Bernien, H., Dreau, A.E., Reiserer, A., Kalb, N., Blok, M.S., Ruitenberg, J., Vermeulen, R.F., Schouten, R.N., Abellan, C., et al.: Experimental loophole-free violation of a Belli nequality using entangled electron spins separated by $1.3 \mathrm{~km}$. Nature 526, 682 (2015)

56. Giustina, M., Versteegh, M.A., Wengerowsky, S., Steiner, J., Hochrainer, A., Phelan, K., Steinlechner, F., Kofler, J., Larsson, J.A., Abellan, C., et al.: A significant-loophole-free test of Bell's theorem with entangled photons. Phys. Rev. Lett. 115, 250401 (2015)

57. Shalm, L.K., Meyer-Scott, E., Christensen, B.G., Bierhorst, P., Wayne, M.A., Stevens, M.J., Gerrits, T., Glancy, S., Hamel, D.R., Allman, M.S., et al.: A strong loophole-free test of local realism. Phys. Rev. Lett. 115, 2504 (2015)

58. Aspect, A.: Trois tests expérimentaux des inégalités de Bell par mesure de corrélation de polarisation de photons, thése d' Etat, Orsay (1983)

59. Adenier, G.: A. Khrennikov, Anomalies in experimental data for the EPR-Bohm experiment: Are both classical and quantum mechanics wrong? arXiv:quant-ph/0607172

60. Valente, M.B.: Bohr's quantum postulate and time in quantum mechanics. philsci-archive.pitt.edu/8335/1/Bohr's $s_{q}$ uantum ostulate $_{a}$ nd $_{t}$ ime $_{i} n_{q}$ uantum $_{m}$ echanics.pdf.

61. von Neuman, J.: Mathematical Foundations of Quantum Mechanics. Princeton University Press, Princenton (1955)

Publisher's Note Springer Nature remains neutral with regard to jurisdictional claims in published maps and institutional affiliations. 